\title{
(6) OPEN ACCESS \\ Estimating the weight of ethnically diverse children attending an Australian emergency department: a prospective, blinded, comparison of age-based and length-based tools including Mercy, PAWPER and Broselow
}

\author{
Fenton O'Leary, ${ }^{1,2}$ Blessy John-Denny, ${ }^{1,2}$ Kathryn McGarvey, ${ }^{2}$ Alexandra Hann, ${ }^{1}$ \\ loannis Pegiazoglou, ${ }^{1,2}$ Jennifer Peat ${ }^{3}$
}

\begin{abstract}
${ }^{1}$ Emergency Department, The Children's Hospital at Westmead, Westmead, New South Wales, Australia 2Disciplines of Emergency Medicine and Paediatrics and Child Health, Sydney Medical School, The University of Sydney, Sydney, New South Wales, Australia

${ }^{3}$ Australian Catholic University, Sydney, New South Wales, Australia
\end{abstract}

Correspondence to Dr Fenton O'Leary, Emergency Department. The Children's Hospital at Westmead, Locked Bag 4001, Westmead, NSW 2145, Australia; fenton.oleary@health.nsw. gov.au

Received 21 March 2016 Revised 1 September 2016 Accepted 3 September 2016 Published Online First 31 October 2016

\section{ABSTRACT}

Objective To prospectively compare the actual weights of Australian children in an ethnically diverse metropolitan setting with the predicted weights using the Paediatric Advanced Weight Prediction in the Emergency Room (PAWPER) tape, Broselow tape, Mercy system and calculated weights using the updated Advanced Paediatric Life Support (APLS), Luscombe and Owens and Best Guess formulae.

Methods A prospective, cross-sectional, observational, blinded, convenience study conducted at the Children's Hospital at Westmead Paediatric Emergency Department in Sydney, Australia. Comparisons were made using BlandAltman plots, mean difference, limits of agreement and estimated weight within $10 \%$ and $20 \%$ of actual weight. Results 199 patients were enrolled in the study with a mean actual weight of $27.2 \mathrm{~kg}$ (SD 17.2). Length-based tools, with or without body habitus adjustment, performed better than age-based formulae. When measuring estimated weight within 10\% of actual weight, PAWPER performed best with 73\%, followed by Mercy (69\%), PAWPER with no adjustment (62\%), Broselow (60\%), Best Guess (47\%), Luscombe and Owens (41\%) and revised APLS (40\%). Mean difference was similar across all methods ranging from $0.4 \mathrm{~kg}(0.0$, $0.9)$ for Mercy to $-2.2 \mathrm{~kg}(-3.5,-0.9)$ for revised APLS. Limits of agreement were narrower for the lengthbased tools (-5.9, 6.8 Mercy; -8.3, 5.6 Broselow; -9.0, 7.1 PAWPER adjusted; -12.1, 9.2 PAWPER unadjusted) than the age-based formulae $(-18.6,17.4$ Best Guess; -19.4, 15.1 revised APLS, -21.8, 17.7 Luscombe and Owens).

Conclusion In an ethnically diverse population, lengthbased methods with or without body habitus modification are superior to age-based methods for predicting actual body weight. Body habitus modifications increase the accuracy and precision slightly.

\section{INTRODUCTION}

An accurate weight is pivotal in the resuscitation of CrossMark To cite: O'Leary $F$, JohnDenny B, McGarvey K, et al. Arch Dis Child

2017;102:46-52. a critically unwell child presenting to an emergency department. ${ }^{12}$ In trauma and resuscitation settings there are limitations in acquiring actual weights from calibrated scales and parents are often not present to provide this information. Visual estimation of the child's weight by healthcare workers is prone to error. ${ }^{3}$ Both overestimation and
What is already known on this topic?

- The estimation of weight for critically ill children is essential for safe drug-prescribing and selecting the correct-sized equipment.

- Many age-based and length-based tools exist.

- Estimation tools may not be accurate with ethnically diverse or different populations.

\section{What this study adds?}

- Confirmation that children attending emergency departments are ethnically diverse.

- Length-based tools, with or without body habitus adjustment are superior to age-based formulae.

- The Paediatric Advanced Weight Prediction in the Emergency Room tape, with body habitus adjustment, is recommended as a length-based estimation tool where age is unknown.

underestimation of a child's weight may lead to suboptimal medication dosing and related morbidity or mortality. ${ }^{4}$

The local population to our emergency department is ethnically diverse. ${ }^{5}$ Our concern was that current weight estimation tools may not correctly estimate weights of patients who are either larger or smaller than 'average'. Age-based tools have been shown to perform poorly with Pacific Islander, Maori, Aboriginal and Torres Strait Islanders, South East Asian, Kenyan and Korean children as well as those with medical conditions such as Down's Syndrome. ${ }^{6-11}$ Even updated tools such as the 'updated APLS' from the fifth edition handbook have been shown to perform worse than the original tool in Filipino children. ${ }^{12} 13$ The Broselow tape is the standard length-based tool and performs better than age-based methods; however, there are concerns that this method may still be inaccurate and that other methods based on body habitus or mid-arm circumference may be more accurate. ${ }^{8} 1114$

We conducted a literature review and identified two validated, length-based tools, the Paediatric 
Figure 1 Images showing the demonstration of the Paediatric Advanced Weight Prediction in the Emergency Room (PAWPER) tape.

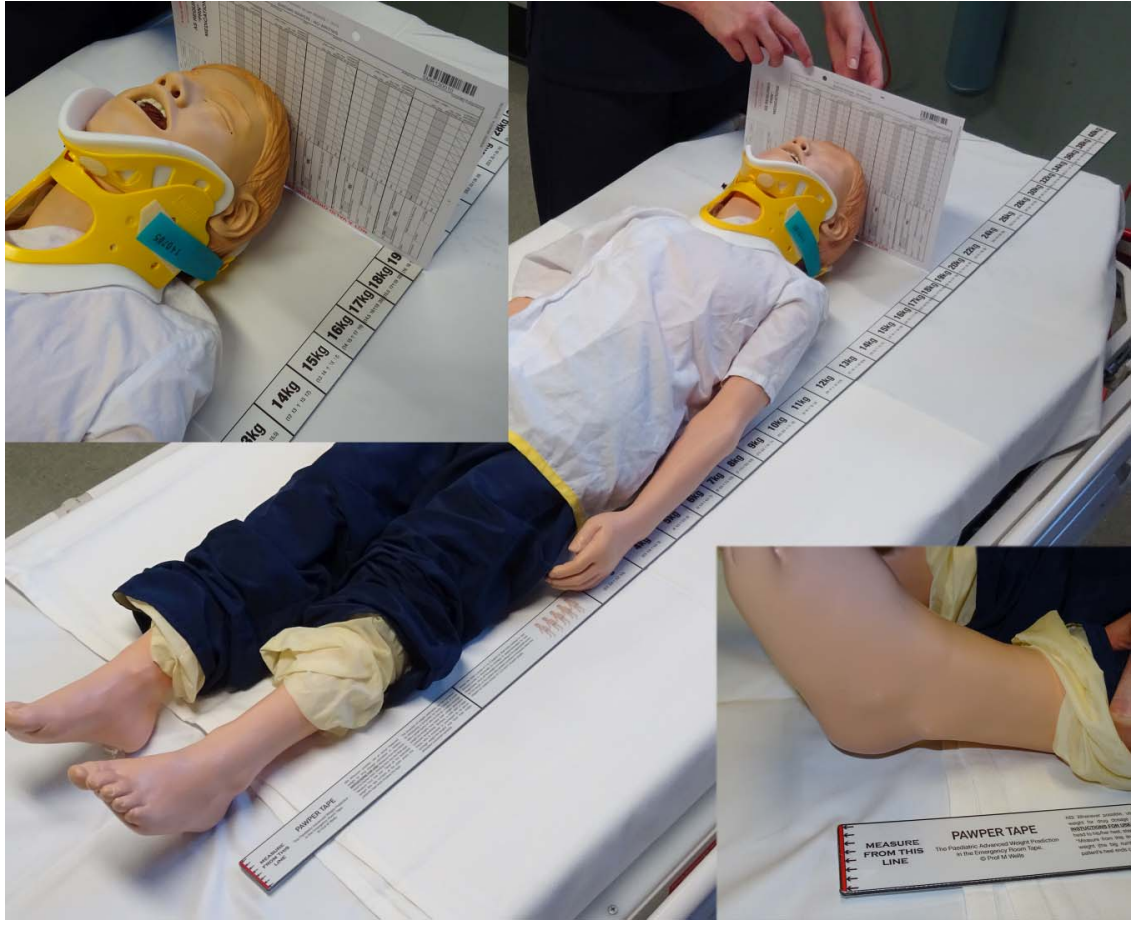

Advanced Weight Prediction in the Emergency Room (PAWPER) tape (figure 1) 15 $^{16}$ and the Mercy method, that modified weight estimations using body habitus. ${ }^{17-19}$

The aim of this study was to prospectively compare the actual weights of Australian children in an ethnically diverse metropolitan setting with the predicted weights using the PAWPER tape, Broselow tape, Mercy system and calculated weights using the updated APLS, Luscombe and Owens and Best Guess formulae. 132021

\section{METHODS}

\section{Study design and setting}

A prospective, cross-sectional, observational, blinded, convenience study was conducted at the Children's Hospital at Westmead Paediatric Emergency Department (PED) in Sydney, Australia, from January to March 2015. The department has over 50000 attendances annually. The study was approved by the Human Research Ethics Committee of the Sydney Children's Hospital Network.

\section{Study participants}

The study participants are convenience sample of infants and children and their siblings, attending the PED during the study period. Patients eligible for inclusion were those aged 0-14 years who already had care initiated by a doctor or nurse practitioner. Appropriate verbal permission was obtained from parents and patients, where applicable, using a standardised information sheet. Exclusion criteria were children: aged $\geq 15$ years, requiring acute resuscitation, known or thought to be pregnant, with suspected or apparent limb deformities or who are unable to be positioned for the actual weight estimation. Uncooperative younger children were also excluded, even if parents consented.

\section{Data collection and study protocol}

Eligible patients were enrolled when two trained data collectors were available. Data were collected by three doctors and four nurses in the PED who had been trained in the use of the weight estimation tools. The two investigators were blinded to each other's measurements, with one measuring the actual weight of the child and the second estimating the weight using the three tools. Aged-based calculations of weight were calculated at the data analysis stage using the following formulae.

The updated APLS weight was computed as follows for ages $0-12$ years:

- Infants $0-12$ months: weight $(\mathrm{kg})=(0.5 \times$ age in months $)+4$

- Children 1-5 years: weight $(\mathrm{kg})=(2 \times$ age in years $)+8$

- Children 6-12 years: weight $(\mathrm{kg})=(3 \times$ age in years $)+7$ Luscombe and Owens was computed as follows for ages 1-14 years: weight $(\mathrm{kg})=(3 \times$ age $)+7$

Best Guess weight was computed as follows:

- Infants 1-11 months: weight $(\mathrm{kg})=($ age in months +9$) / 2$

- Children 1-4 years: weight $(\mathrm{kg})=2 \times(\mathrm{age}+5)$

- Children 5-14 years: weight $(\mathrm{kg})=4 \times$ age

In addition to the standard demographics of age and sex, parents were asked to verbally define their ethnicity.

Actual weight was measured with the patient in light clothing, defined as one layer of clothes, on a digital scale to the nearest $0.1 \mathrm{~kg}$, with heavy outer clothing and shoes removed. Calibrated scales were used for patients capable of standing (AND HV-150 KA1, Colonial weighing, Campbellfield, Australia) and a supine scale for infants (SECA Mod 727, Hamburg, Germany).

The second investigator (blinded to age and actual weight) first estimated patient body habitus according to the PAWPER method described in table 1 and assigned a habitus score. They then estimated the patients' weight using the Broselow Paediatric Emergency Tape (2007, Edition B, Armstrong Medical Industries, Illinois, USA), PAWPER tape followed by mid-arm circumference and mid-humeral length for the Mercy tape, according to the designers instructions. ${ }^{15} 22$

The primary outcome was the estimated weight for each of the estimation tools compared with actual weight. Secondary outcomes were demographic information. 
Table 1 Body habitus scoring of PAWPER tape

\begin{tabular}{ll}
\hline Habitus score & Patient characteristic \\
\hline 1 & Very thin, somewhat wasted or tiny frame \\
2 & Thin, petite, slim or small frame \\
3 & Average body fat and frame size \\
4 & Heavy, chubby, overweight or large frame \\
5 & Fat, significantly overweight or obese \\
\hline PAWPER, Paediatric Advanced Weight Prediction in the Emergency Room.
\end{tabular}

\section{Data analysis}

Data were analysed using SPSS (V.21.0; IBM, USA). For each child, estimated weight was calculated from age or length for each reported weight estimation method and was summarised by age group. Percentage weight was computed as estimated weight divided by measured weight and multiplied by 100 . This was used to assess the number of children whose percentage weight was within $10 \%$ and $20 \%$, which is in the range $-10 \%$ to $10 \%$ and $-20 \%$ to $20 \%$.

Level of agreement was determined by applying the Bland-Altman approach, with plots modified for the $\mathrm{x}$ axis as described by Krouwer. ${ }^{23-25}$ Mean difference (estimated weightactual weight) was calculated with respective 95\% limits of agreement and 95\% CIs around each of these values. Bland-Altman plots were performed to allow visual comparison of the different methods. Accuracy refers to the mean difference and precision to the limits of agreement as described by Cecconi et al. ${ }^{26}$

Following the recommendations of Bland a sample size of 200 was chosen to provide $95 \%$ CIs of $\pm 0.24 \times$ the SD of the differences between measurements by the two methods. ${ }^{27} \mathrm{~A}$ sensitivity analysis was obtained on the overall data, removing the contribution of children aged 11-14 years, to determine the influence of the smaller numbers of patients able to be measured by the length-based tapes in this age group.

\section{Results}

A total of 199 patients were enrolled, with a mean actual weight of $27.2 \mathrm{~kg}$ (SD 17.2). No patients were excluded after enrolment. A total of 109 patients (54\%) were male. There were 170 patients whose length was within the range of the PAWPER tape and 160 patients whose length was within the range of the Broselow tape. Table 2 describes the mean weight by age, with numbers of patients in each age bracket. Self-reported ethnicity is described in table 3.

The results of the mean difference, limits of agreement and percentage within $10 \%$ and $20 \%$ are shown in table 4 . The Bland-Altman plots are shown in figure 2. Overall, the age-based methods performed worse than the length and body habitus methods, with aged-based tools having 40.4\%-47.2\% of estimated weights within $10 \%$ of actual, compared with $60 \%-$ $72.9 \%$ for the others. Likewise, although the mean difference for all methods was quite similar, the limits of agreement were narrower for the length-based and habitus-based methods making them more precise.

Of the age-based prediction tools, Best Guess was the most accurate, although all three methods produced similar results. The Mercy method was the most accurate with an overall mean difference of only $0.4 \mathrm{~kg}$; it was the most precise and had $69 \%$ of estimated weights within $10 \%$ of actual weight. The PAWPER tape with body habitus adjustment performed better
Table 2 Results-mean weight by age

\begin{tabular}{lcl}
\hline Age (years) & No. & $\begin{array}{l}\text { Weight } \mathbf{( k g}) \\
\text { Median (IQ range) }\end{array}$ \\
\hline 0 & 18 & $8.5(1.9)$ \\
1 & 14 & $12.2(1.9)$ \\
2 & 18 & $14.0(3.7)$ \\
3 & 16 & $15.1(3.2)$ \\
4 & 16 & $17.5(4.3)$ \\
5 & 14 & $20.5(4.5)$ \\
6 & 11 & $24.1(10.6)$ \\
7 & 15 & $26.2(7.9)$ \\
8 & 20 & $31.2(3.1)$ \\
9 & 9 & $36.0(11.8)$ \\
10 & 13 & $37.7(14.2)$ \\
11 & 7 & $38.7(41.0)$ \\
12 & 12 & $43.6(14.0)$ \\
13 & 11 & $53.0(20.0)$ \\
14 & 5 & $50.5(17.3)$ \\
\hline
\end{tabular}

Table 3 Self-reported ethnicity

\begin{tabular}{lc}
\hline Parent-identified ethnic group & Total no. (\%) \\
\hline Anglo-Saxon & $65(32.7 \%)$ \\
Asian & $31(15.6 \%)$ \\
Middle Eastern & $30(15.1 \%)$ \\
Indian Subcontinent & $19(9.5 \%)$ \\
African & $11(5.5 \%)$ \\
Pacific Islander & $9(4.5 \%)$ \\
Aboriginal or Torres Strait Islander & $8(4.0 \%)$ \\
Mediterranean & $4(2.0 \%)$ \\
Hispanic & $2(1.0 \%)$ \\
Mixed & $20(10.0 \%)$ \\
\hline
\end{tabular}

than with no adjustment and is overall almost as accurate as Mercy with a mean difference of $-1.0 \mathrm{~kg}$ and has the highest estimated weights within $10 \%$ of actual weight (72.9\%). A sensitivity analysis, excluding those patients aged 11-14 years, showed PAWPER tape to be the most precise tool, although the limits of agreement improved across all methods.

\section{DISCUSSION}

This study has demonstrated that for patients unable to be weighed in an ethnically diverse PED, the length-based and body habitus-based tools perform better than age-based tools. Overall, the length-based tools had much narrower limits of agreement and were therefore more precise. With the age-based tools, there was very little difference between limits of agreement or mean difference, suggesting similar accuracy and precision. With the length-based tools, the accuracy and precision was similar also. The Mercy system was more accurate in children within age group $6-10$ and $11-14$ years. For the age group of 11-14 years, the mean difference of $-0.1 \mathrm{~kg}$, the narrow $95 \%$ CIs of -2.0 to 1.8 , and the relatively narrow limits of agreement of -11 to 10.9 contrast with the other methods and show its utility in the older aged children who have wider weight distributions. 


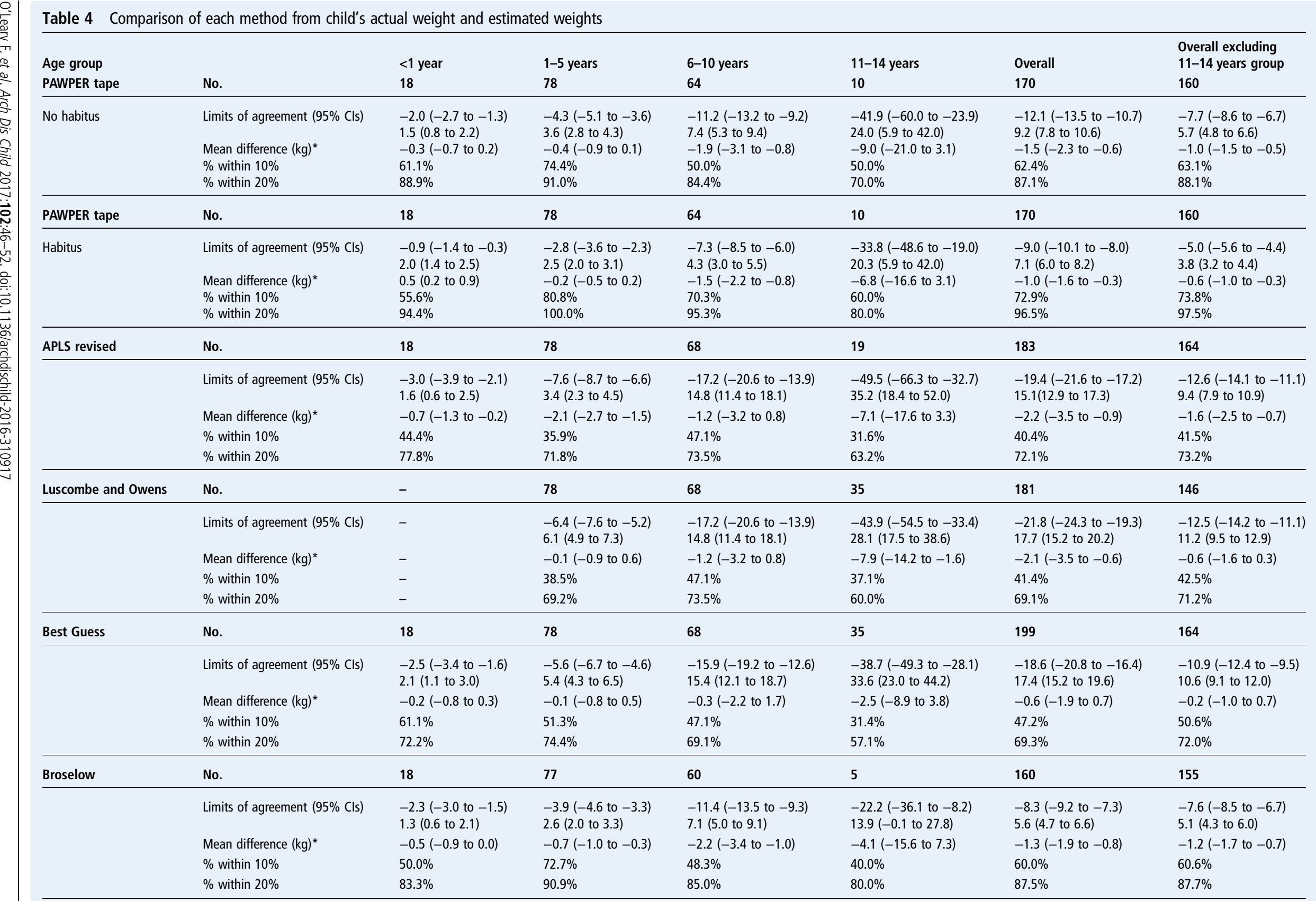




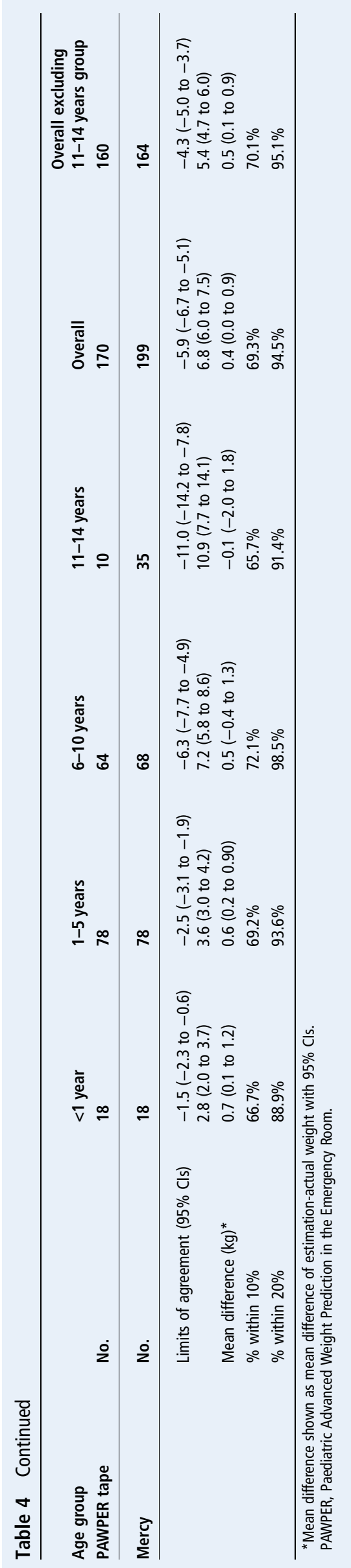

Using estimated weight within $10 \%$ of actual weight as a comparison, the PAWPER tape with adjustment performed best with $73 \%$ overall within $10 \%$, consistent with previous studies. ${ }^{7} 17$ Some authors have rated this measure as the primary method for comparing weight estimations. ${ }^{28}$ In a study by Garcia et al, PAWPER performed less well with only $64 \%$ within $10 \%$ and the authors stated that education of evaluators on the definition of body habitus may have been a factor. ${ }^{16}$ This may also be why in the under-12-month category in this study the PAWPER with adjustment tool has a lower percentage within 10\% than the unadjusted PAWPER estimates (56\% vs 61\%). Further research is required to determine the factors that influence body habitus score allocation, such as patient position, as well as conscious and unconscious biases.

Since the completion of the study, the APLS sixth edition handbook has been released and it is interesting that they have reverted back to tabulated estimates with more age points. This may reflect the previous literature and the results of this study showing that age-based formulae poorly estimate actual weight. 792930

A recent systematic review by Young and Korotzer ${ }^{28}$ concluded that age-based and length-based methods without habitus adjustment tended to predict ideal body weight. Although beyond the scope of this paper, clinicians need to consider whether an actual or ideal estimate of weight is required as some drugs may be optimally administered at ideal body weight. $^{31}$

When comparing the length and habitus tools, the Broselow tape has an advantage of providing a whole ecosystem of preselected drugs and equipment, which may be an advantage in an emergency. This may offset the slightly inferior results, although up to $60 \%$ of patients may be misclassified by colour band resulting in inappropriate drug doses or equipment choice. ${ }^{32}$ The Mercy tape performed well in this study and had the advantage of not being limited by patient length and therefore able to estimate in all patients. The study investigators used the tool in the way described by the designers. ${ }^{17}$ However, this method of using the tool with the patient upright and the arm hanging down is not appropriate in a trauma patient who is supine. The posterior border of the acromion process can be difficult to access in this position. Further research is required to validate the use of this tool in supine patients and to determine if this high degree of accuracy and precision is maintained.

The self-reported ethnicity described in table 4 is consistent with the local population and we feel that the 199 patients were a representative sample of those attending the PED, although our local population may not be representative of other populations. Another limitation of the study could be the influence of the smaller numbers of older children and their influence on the overall results; however, the sensitivity analysis failed to show a difference. Study investigators may have had different perceptions of normal and abnormal body habitus which may have influenced the results of the PAWPER tape. This study was a convenience study and this sampling method could have introduced bias into the sample chosen for inclusion as could the method of only using the trained investigators to perform the measurements. Further studies may be required with larger numbers of investigators to determine the degree of intra-observer and inter-observer variability with the new tools.

\section{CONCLUSION}

In an ethnically diverse population, length-based methods, with or without body habitus modification, are superior to age-based 

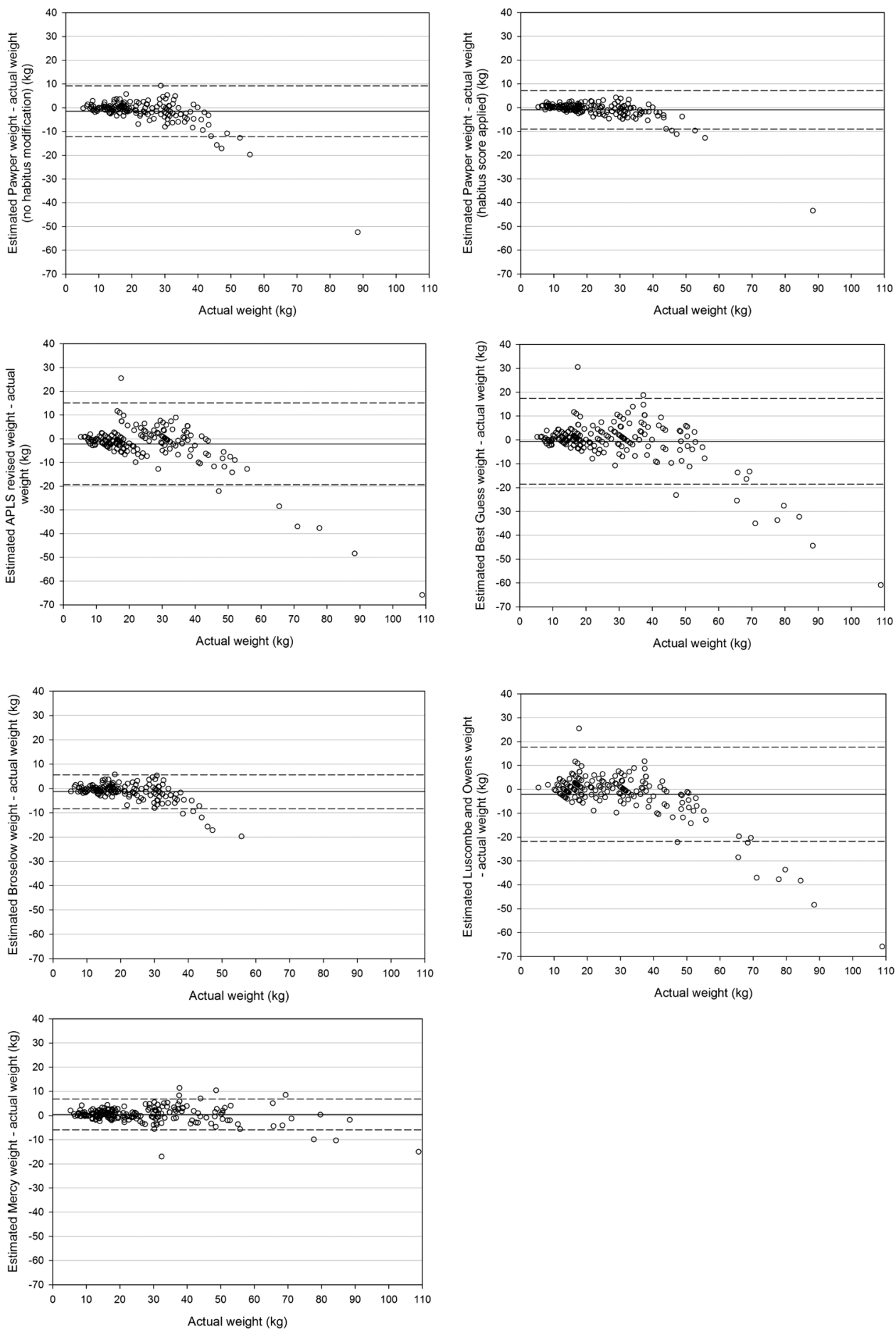

Figure 2 Bland-Altman plots showing predicted versus actual weight for each weight estimation method. Continuous line represents the mean difference and dashed line represents the limits of agreement.

methods for predicting actual body weight. Body habitus modifications increase the accuracy and precision slightly. Balancing estimated weight results with ease of use, the PAWPER tape with body habitus adjustment is recommended overall. Where a patient is too tall for PAWPER and their age is unknown, then Mercy should be used.

Acknowledgements The authors would like to thank Professor Mike Wells for providing information regarding the PAWPER tape and Susan Abdel-Rahman for providing information on the Mercy system.

Competing interests None declared.
Ethics approval Human Research Ethics Committee of Sydney Children's Hospitals Network.

Provenance and peer review Not commissioned; externally peer reviewed.

Data sharing statement All outcome data have been included in the paper. Authors will consider access to raw study data on request.

Open Access This is an Open Access article distributed in accordance with the Creative Commons Attribution Non Commercial (CC BY-NC 4.0) license, which permits others to distribute, remix, adapt, build upon this work non-commercially, and license their derivative works on different terms, provided the original work is properly cited and the use is non-commercial. See: http://creativecommons.org/ licenses/by-nc/4.0/ 


\section{REFERENCES}

1 Luten R. Error and time delay in pediatric trauma resuscitation: addressing the problem with color-coded resuscitation aids. Surg Clin North Am 2002;82:303-14, vi.

2 Hofer CK, Ganter M, Tucci M, et al. How reliable is length-based determination of body weight and tracheal tube size in the paediatric age group? The Broselow tape reconsidered. Br J Anaesth 2002;88:283-5.

3 Harris M, Patterson J, Morse J. Doctors, nurses, and parents are equally poor at estimating pediatric weights. Pediatr Emerg Care 1999;15:17-18.

4 Kaushal R, Bates DW, Landrigan C, et al. Medication errors and adverse drug events in pediatric inpatients. JAMA 2001;285:2114-20.

5 Statistics. ABo. Census of Population and Housing 2011. http://profile.id.com.au/ wsroc/ancestry?BMID $=40 \&$ WebID $=180$

6 Allison N, Norton I. Emergency weight estimation in Aboriginal and Torres Strait Islander children in the Northern Territory: are the current methods accurate? Emerg Med Australas 2014;26:487-93.

7 Britnell S, Koziol-McLain J. Weight estimation in paediatric resuscitation: a hefty issue in New Zealand. Emerg Med Australas 2015;27:251-6.

8 Erker CG, Santamaria M, Moellmann M. Size does matter--age-related weight estimation in "tall $\mathrm{n}^{\prime}$ thin" and "tiny $\mathrm{n}$ ' thick" children and a new habitus-adapted alternative to the EPLS-formula. Resuscitation 2014;85:1174-8.

9 House DR, Ngetich E, Vreeman RC, et al. Estimating the weight of children in Kenya: do the Broselow tape and age-based formulas measure up? Ann Emerg Med 2013:61:1-8.

10 Talib NJ, Rahm G, Abdel-Rahman SM. Estimating weight in children with down syndrome. Glob Pediatr Health 2015;2:2333794X14568450.

11 Theron L, Adams A, Jansen K, et al. Emergency weight estimation in Pacific Island and Maori children who are large-for-age. Emerg Med Australas 2005;17:238-43.

12 Young TP, Washington O, Flanery A, et al. Comparison of the finger counting method, the Broselow tape and common weight estimation formulae in Filipino children after Typhoon Haiyan. Emerg Med Australas 2015;27:239-44.

13 Group. ALS. Advanced paediatric life support: the practical approach. 5th edn. Oxford: Wiley-Blackwell, 2012

14 Cattermole GN, Leung PY, Mak PS, et al. Mid-arm circumference can be used to estimate children's weights. Resuscitation 2010:81:1105-10.

15 Wells M, Coovadia A, Kramer E, et al. The PAWPER tape: a new concept tape-based device that increases the accuracy of weight estimation in children through the inclusion of a modifier based on body habitus. Resuscitation 2013;84:227-32.

16 Garcia CM, Meltzer JA, Chan KN, et al. A Validation Study of the PAWPER (Pediatric Advanced Weight Prediction in the Emergency Room) Tape-a new weight estimation tool. J Pediatr 2015;167:173-7.e1.
17 Abdel-Rahman SM, Paul IM, James LP, et al. Evaluation of the Mercy TAPE: performance against the standard for pediatric weight estimation. Ann Emerg Med medicine 2013;62:332-9.e6

18 Dicko A, Alhousseini ML, Sidibe B, et al. Evaluation of the Mercy weight estimation method in Ouelessebougou, Mali. BMC Public Health 2014;14:270.

19 Abdel-Rahman SM, Ridge A, Kearns GL. Estimation of body weight in children in the absence of scales: a necessary measurement to insure accurate drug dosing. Arch Dis Child 2014:99:570-4

20 Luscombe M, Owens B. Weight estimation in resuscitation: is the current formula still valid? Arch Dis Child 2007:92:412-15.

21 Kelly AM, Kerr D, Clooney M, et al. External validation of the Best Guess formulae for paediatric weight estimation. Emerg Med Australas 2007:19:543-6.

22 Abdel-Rahman SM, Ahlers N, Holmes A, et al. Validation of an improved pediatric weight estimation strategy. J Pediatr Pharmacol Ther 2013;18:112-21.

23 Bland JM, Altman DG. Statistical methods for assessing agreement between two methods of clinical measurement. Lancet 1986;1:307-10.

24 Krouwer JS. Why Bland-Altman plots should use $X$, not $(Y+X) / 2$ when $X$ is a reference method. Stat Med 2008;27:778-80.

25 Hanneman SK. Design, analysis, and interpretation of method-comparison studies. AACN Adv Crit Care 2008;19:223-34.

26 Cecconi M, Rhodes A, Poloniecki J, et al. Bench-to-bedside review: the importance of the precision of the reference technique in method comparison studies-with specific reference to the measurement of cardiac output. Crit Care 2009;13:201.

27 Bland JM. How can I decide the sample size for a study of agreement between two methods of measurement? 2004 (updated 12 January 2004; cited 28 June 2016). https://www-users.york.ac.uk/ mb55/meas/sizemeth.htm

28 Young KD, Korotzer NC. Weight estimation methods in children: a systematic review. Ann Emerg Med 2016 Apr 14. pii: S0196-0644(16)00147-5. doi: 10.1016/j. annemergmed.2016.02.043. [Epub ahead of print]

29 Group. ALS. Advanced paediatric life support: a practical approach to emergencies. 6th edn. Oxford: Wiley-Blackwell, 2016.

30 Geduld H, Hodkinson PW, Wallis LA. Validation of weight estimation by age and length based methods in the Western Cape, South Africa population. Emerg Med J 2011;28:856-60

31 Dieckmann RA. The dilemma of paediatric drug dosing and equipment sizing in the era of patient safety. Emerg Med Australas 2007;19:490-3.

32 Graves L, Chayen G, Peat J, et al. A comparison of actual to estimated weights in Australian children attending a tertiary children's' hospital, using the original and updated APLS, Luscombe and Owens, Best Guess formulae and the Broselow tape. Resuscitation 2014;85:392-6. 